\title{
Spatio-temporal patterns in the Hantavirus infection
}

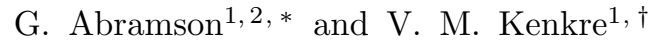 \\ ${ }^{1}$ Center for Advanced Studies and Department of Physics and Astronomy, \\ University of New Mexico, Albuquerque, New Mexico 87131 \\ ${ }^{2}$ Centro Atómico Bariloche and CONICET, 8400 S. C. de Bariloche, Argentina
}

(Dated: November 1, 2018)

\begin{abstract}
We present a model of the infection of Hantavirus in deer mouse, Peromyscus maniculatus, based on biological observations of the system in the North American Southwest. The results of the analysis shed light on relevant observations of the biological system, such as the sporadical disappearance of the infection, and the existence of foci or "refugia" that perform as reservoirs of the virus when environmental conditions are less than optimal.

PACS numbers: 87.19.Xx, 87.23.Cc, 05.45.-a
\end{abstract}

\section{INTRODUCTION}

Hantaviruses are infectious agents carried by rodents throughout the whole world [1, 2, 3. Some of them are able to cause severe disease in humans, with a mortality rate of around $50 \%$, as in the case of the Hantavirus Pulmonary Syndrome (HPS) caused by the Sin Nombre Virus in the North American Southwest, or the Andes Virus in Patagonia. With few exceptions, each hantavirus is associated with a single primary rodent host species, chronically infected, and infects humans that come into contact with it or its excreta. Sin Nombre Virus is primarily carried by the deer mouse, Peromyscus maniculatus, the most numerous mammal in North America, prevalent throughout the region. It was the cause of an outbreak of fatal pulmonary disease in the Southwest of the United States in 1993, when the virus was first isolated and described. Since then, a great effort has been devoted to understand the nature of the virus reservoir, its temporal and spatial dynamics, and its relation to the human population, in an attempt to ultimately identify and predict the risk of the disease.

Needless to say, a complete mathematical description of the dynamics of the biological system, comprising the virus, the mice, the humans and the environment, is a daunting task. The goal of the present investigation is much less ambitious. From the biological complexities we extract a few major components centered on the basic ecological and epidemiological features of the mice population. As the motivation for our analysis we choose two observed characteristics of the disease. Both arise from the fact that environmental conditions strongly affect the dynamics and persistence of the infection. One of them, a temporal characteristic, is the reported observation that the infection can completely disappear from a population of mice if environmental conditions are inadequate, only to reappear sporadically or when conditions change [3, 4, 5]. The other, a spatial characteristic, is

\footnotetext{
*Electronic address: abramson@cab.cnea.gov.ar
}

${ }^{\dagger}$ Electronic address: kenkre@unm.edu that there are indications of "focality" of the infection in "reservoir" populations [3, 6]; as environmental changes occur, these "refugia" [7] of the reservoir can expand or contract, carrying the infection to other places.

The model we introduce incorporates the decay by death of the mice population, the spread of the infection through their interaction, the increase by birth and effect of the environment to stabilize the population, and also their movement as a process of diffusion. We begin in Section II by first omitting the last feature (the movement from one location to another), motivating the different dynamical mechanisms, and obtaining some basic results including the observed temporal behavior. We proceed in Section III to perform a spatial extension of the model to include movement and obtain results relating to the refugia. A summary is given in the final section.

\section{BASIC MODEL OF MOUSE POPULATION}

We can incorporate the basic ingredients of the biological system in a model of the mouse population only [8] We suppose that the whole population is composed of two classes of mice, susceptible and infected, represented by $M_{S}$ and $M_{I}$ respectively. Sex and age composition of the population are disregarded in this basic model. The temporal evolution of $M_{S}$ and $M_{I}$ contains two basic ingredients: the contagion of the infection, that converts susceptible into infected, and a population dynamics independent of the infection:

$$
\begin{aligned}
& \frac{d M_{S}}{d t}=b M-c M_{S}-\frac{M_{S} M}{K}-a M_{S} M_{I}, \\
& \frac{d M_{I}}{d t}=-c M_{I}-\frac{M_{I} M}{K}+a M_{S} M_{I},
\end{aligned}
$$

where $M_{S}$ and $M_{I}$ are the populations (or densities) of susceptible and infected mice, respectively, and $M(t)=$ $M_{S}(t)+M_{I}(t)$ is the total population of mice. The motivation for the terms in Eqs. (112) follows.

Births: $b M$ represents births of mice, all of them born susceptible, at a rate proportional to the total density, since all mice contribute equally to the procreation [3]. 
Deaths: $c$ represents the rate of depletion by death for natural reasons, proportional to the corresponding density. If necessary, separate rates $c_{S}$ and $c_{I}$ could be introduced for the susceptible and infected populations respectively.

Competition: $-M_{S, I} M / K$ represent a limitation process in the population growth, due to competition for shared resources. Each is proportional to the probability of an encounter of a pair formed by one mouse of the corresponding class, susceptible or infected, and one mouse of any class (since every mouse, either susceptible or infected, has to compete with the whole population). $K$ is a "carrying capacity," characterizing in a simplified way the capacity of the medium to maintain a population of mice. Higher values of carrying capacity represent a higher availability of water, food, shelter and other resources that mice can use to thrive [9].

Infection: $a M_{I} M_{S}$ represents the number of susceptible mice that get infected, due to an encounter with an infected (and consequently infectious) mouse, at a rate $a$ that we assume constant. More elaborate models could incorporate a density dependence on $a$, for example due to an increased frequency of fights, during which contagion occurs through bites, when the density is too high and the population feels overcrowded 顿. The infection is chronic, infected mice do not die of it, and infected mice do not lose there infectiousness probably for their whole life [3, 6]. For these reasons, this single term adequately describes the infection dynamics of the two subpopulations.

The sum of the two equations (1,2) reduces to a single equation for the whole population of logistic form:

$$
\frac{d M}{d t}=(b-c) M\left(1-\frac{M}{(b-c) K}\right) \text {. }
$$

Logistic growth has been observed in laboratory populations of Peromyscus [10], and is a well established metaphor of the dynamics of a self limitating population [9].

There are four parameters that characterize the system (1.22), viz. $a, b, c$ and $K$. Of these, we will choose $K$ as a control parameter of the dynamics, since it is the one that best represents the influence of the environment.

The system (1,2) has four equilibria. Two of them are irrelevant to the present analysis (the null state, which is always unstable, and a state with $M_{I}<0$ for any parameters). The other two equilibria interchange their stability character at a critical value of the carrying capacity, a result that we show in Fig. 11 as a bifurcation diagram. The critical value of the carrying capacity is

$$
K_{c}=\frac{1}{a}\left(\frac{b}{b-c}\right) \text {. }
$$

We can see that the prevalence of the infection can be correlated, through $K$, with the diversity of habitats and other ecological conditions. Thus, a scarcity of resources - that is to say, a low value of $K$ - is accompanied by a lower number of infected mice, as found in

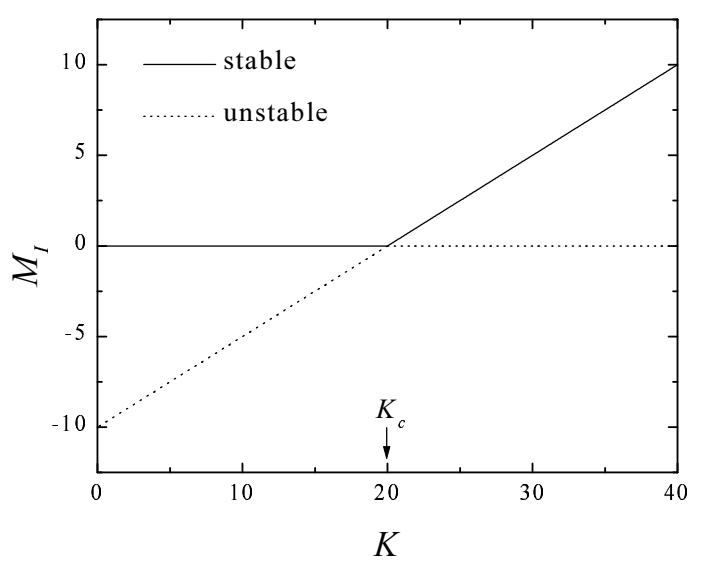

FIG. 1: Bifurcation diagram of the density of infected mice $M_{I}$, as a function of the carrying capacity $K$. Model parameters are: $a=0.1, b=1, c=0.5$.

field studies such as [3, 6, 11]. Moreover, for values of $K$ below the threshold $K_{c}$ the number of infected animals is effectively zero, a fact that has also been observed in the field (see for example [3, 4, 5]). That is, if temporarily the ecological conditions at a place in the landscape get adverse for the mice (because of a drought, for example) the infection can drop to zero. Correspondingly, when conditions improve again the infection reappears. The density of infected mice can even display a dramatic increase with respect to previous years, if a rare climatic event such as El Niño Southern Oscillation brings enhanced precipitation and the consequent increase in edible resources for the mice. An El Niño event in 1991-1992, precisely, preceded the outbreak of HPS in 1993 in the Southwest [12].

Figure 2 shows a simulation of such events, within the context of the present model. A time-dependent carrying capacity is shown in Fig. 2 (top), and the corresponding values of the susceptible and infected mice populations, $M_{S}(t)$ and $M_{I}(t)$ respectively, are displayed in Fig. 2 (bottom). We model the carrying capacity with a yearly sinusoidal behavior to emulate seasonal variations. A period of 20 years is shown, during which the carrying capacity oscillates around a value, sometimes above $K_{c}$ (shown as a horizontal line), sometimes below it. Discontinuities in the carrying capacity, some of which are present in Fig. 2 (top), do not necessarily occur in nature, and appear here because we keep the modeling of $K(t)$ at an elementary level, to illustrate the main features of the system. The period marked "a" in Fig. 2 (from years 6 to 8 ) is characterized by values of $K$ below $K_{c}$, and corresponds to very adverse environmental conditions. During these "bad years" the infection level effectively drops to zero, while the population of healthy mice, even if reduced, subsists. A return to "normal" carrying capacities after year 8 produces a very slow recovery of the infected population, which attains again appreciable values after year 11. An extraordinary event on year 17 is marked as 


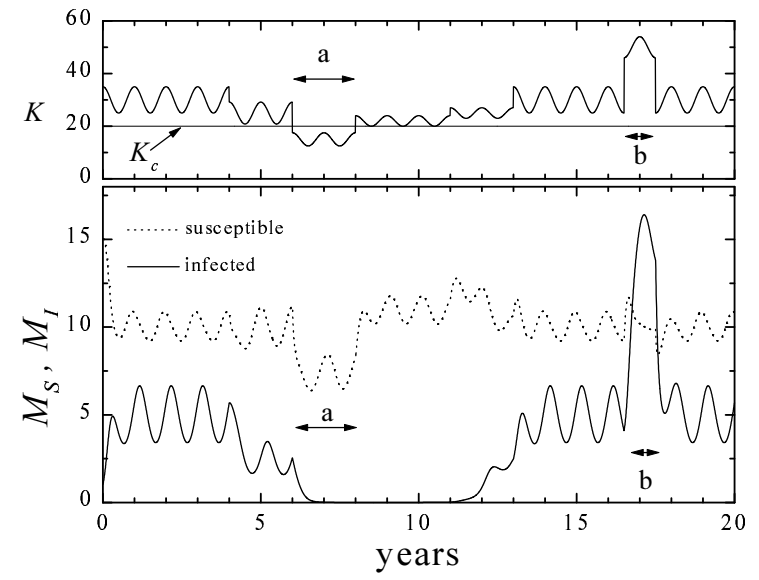

FIG. 2: Temporal evolution of the population of mice (bottom) in a caricature time-dependent carrying capacity (top). Two special events are marked: (a) The carrying capacity is below the $K_{c}$ threshold (shown as a horizontal line). (b) An extraordinary one-year event of greater carrying capacity. Same parameters as in Fig. 11.

"b" in Fig. 2. It corresponds to an increase in the carrying capacity (top), perhaps following an event such as El Niño the year before. These improved environmental conditions are followed by an immediate (if moderate) increase in the population of susceptible mice (bottom, dotted line), and by a slightly delayed outbreak of infection (bottom, full line). An event such as this would appreciably increase the risk for the human population to become infected.

\section{SPATIALLY EXTENDED MODEL}

The range of the deer mice is wide, comprising a diverse landscape with a variety of habitats. This spatial extension and the inhomogeneous way in which it affects local populations can be included in a spatially extended version of the model, where $M_{S}, M_{I}$ and $K$ become functions of a space variable $\mathbf{x}$. Diffusive movement of the mice provide an adequate mechanism of transport, since mice of the genus Peromyscus are known to hold a home range during most of their adult life, occasionally shifting it to nearby locations, in particular if these are vacant [13, 14]. In principle, different diffusion coefficients should be used for susceptible and infected mice. The observation that juvenile animals are the most mobile [4] and that the infection affects mainly adult males [2] certainly supports this. We will choose later, however, for the sake of simplicity of the model, to keep both diffusivities equal. The extended model can be written as:

$$
\frac{\partial M_{S}}{\partial t}=f\left(M_{S}, M_{I}\right)+D_{S} \nabla^{2} M_{S}
$$

$$
\frac{\partial M_{I}}{\partial t}=g\left(M_{S}, M_{I}\right)+D_{I} \nabla^{2} M_{I}
$$

where $f$ and $g$ are the r.h.s. of Eqs. (11) and (2) respectively (and contain the specific form of the spatial dependence $K(\mathbf{x})$ ), and we include separate diffusion coefficients $D_{S}$ and $D_{I}$ for the two classes of mice.

The solution of the system (5.6), and even its stationary solution, may impossible to find, analytically, for an arbitrary function $K(\mathbf{x})$. We describe below some general considerations about stability, followed by numerical solution for x-dependent $K$.

\section{A. Stability of the extended solutions}

Suppose that $M_{S}^{*}(\mathbf{x})$ and $M_{I}^{*}(\mathbf{x})$ are stationary solutions of Eqs. (5),6), i.e. they are solutions of a Laplace equation with nonlinear, space-dependent sources:

$$
\begin{aligned}
\nabla^{2} M_{S} & =-f\left(M_{S}, M_{I}\right) / D_{S}, \\
\nabla^{2} M_{I} & =-g\left(M_{S}, M_{I}\right) / D_{I},
\end{aligned}
$$

found by setting the time derivative of Eqs. (5.6) equal to zero. A perturbation around this equilibrium can be written as:

$$
\begin{aligned}
& M_{S}(\mathbf{x}, t)=M_{S}^{*}(\mathbf{x})+u_{S}(\mathbf{x}, t), \\
& M_{I}(\mathbf{x}, t)=M_{I}^{*}(\mathbf{x})+u_{I}(\mathbf{x}, t) .
\end{aligned}
$$

When the two-component vector $u=\left(u_{S}, u_{I}\right)$ describing the perturbation is inserted into the differential equations (5,6), a linearization around the equilibrium solutions yields

$$
\frac{\partial u(\mathbf{x}, t)}{\partial t}=A(\mathbf{x}) u(\mathbf{x}, t)+D \nabla^{2} u(\mathbf{x}, t),
$$

where $A(\mathbf{x})$ is the linearization of the nonlinear terms of Eqs. (5,6) around the equilibrium, viz.,

$$
A(\mathbf{x})=\left[\begin{array}{cc}
\frac{\partial f}{\partial M_{S}} & \frac{\partial f}{\partial M_{I}} \\
\frac{\partial g}{\partial M_{S}} & \frac{\partial g}{\partial M_{I}}
\end{array}\right]_{\left\{M_{S}^{*}, M_{I}^{*}\right\}},
$$

and $D$ is the $2 \times 2$ diagonal matrix of the diffusivities.

Solutions of Eq. (11) can be looked for in the form of plane waves,

$$
u(\mathbf{x}, t) \sim e^{i \mathbf{k} \cdot \mathbf{x}+\lambda t},
$$

which, in Eq. (11), satisfies:

$$
\left[\lambda I-A(\mathbf{x})+k^{2} D\right] u(\mathbf{x}, t)=0,
$$

where $I$ is the identity matrix. The nontrivial solutions of Eq. 14) will provide a dispersion relation $\lambda\left(k^{2}\right)$, implicitly:

$$
\operatorname{det}\left[\lambda I-A(\mathbf{x})+k^{2} D\right]=0 .
$$




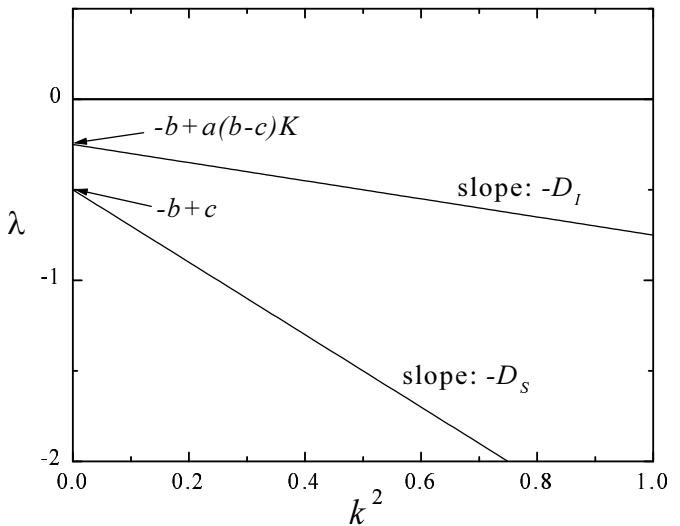

FIG. 3: Dispersion relations between the temporal eigenvalue $\lambda$ and the squared modulus of the wave number of the perturbation, $k^{2}$, for the two homogeneous steady states. Model parameters as in Fig. 1, $K=15, D_{S}=1, D_{I}=0.5$.

In the general situation of $\mathbf{x}$-dependent $K$, it is not possible to proceed further without the knowledge of the equilibria. However, in a system where $K$ does not depend on the space variable, an analytic assessment of the stability of the homogeneous steady states is possible. We have again two relevant steady states: $\left\{M_{S}^{*}=(b-c) K, M_{I}^{*}=0\right\}$ and $\left\{M_{S}^{*}=b / a, M_{I}^{*}=\right.$ $-b / a+(b-c) K\}$. The dispersion relations corresponding to each one of these are easily found from Eq. (15). Those corresponding to the first one (the equilibrium with $M_{I}^{*}=0$ ) are shown in Fig. 3. They provide a direct stability criterion. The slopes of the two lines are determined by the diffusion coefficients only, and as such are always negative. It can be seen that one of the temporal eigenvalues is always negative, provided that $b>c$, which is, obviously, the sensible case in the biological context since otherwise no positive solutions are found. The other eigenvalue is negative provided that $K<K_{c}$, which is the same stability condition found in the nonextended case. Furthermore, when the state becomes unstable, the fastest growing mode of the perturbation (the one with larger $\lambda$ ) is that with $k^{2}=0$, an homogeneous perturbation. Under such conditions, the perturbation eventually drives the system to the other homogeneous steady state, having a nonzero infected population. In this simple model, hence, there are no spatially dependent instabilities to the homogeneous steady state.

\section{B. Refugia}

Certainly, the most interesting situations arise when $K$ exhibits a spatial dependency. This is in fact the case in the field, where $K$ follows the diversity of the landscape. We have analyzed two cases of this situation, by means of a numerical solution of Eqs. (5, 6). The first case is a

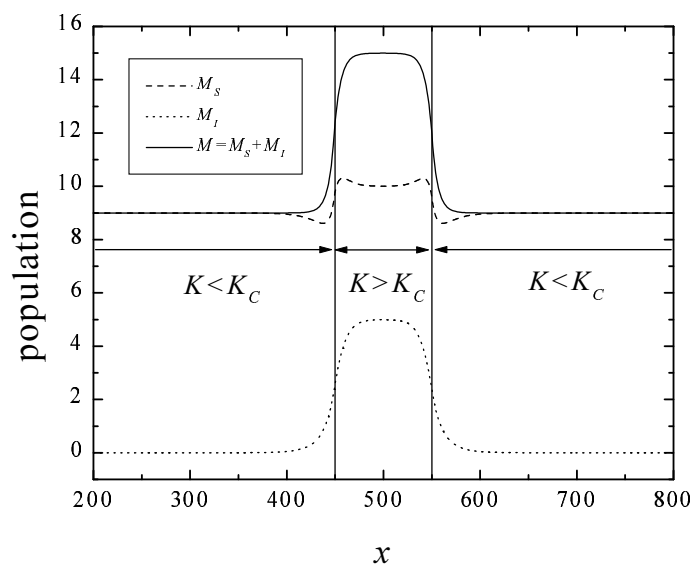

FIG. 4: Stationary solution of the extended model in one dimension. The carrying capacity $K$ consists of a spot of high value, $K>K_{c}$, immersed in a region of lower capacity, $K<K_{C}$. The boundaries are shown as vertical lines. Model parameters as in Fig. 1, $D=20, K=1.5 K_{c}$ in the refugium, $K=0.9 K_{c}$ outside of it.

1-dimensional system, where the profile displayed by the stationary solutions of the populations is readily accessible. The second one is a 2-dimensional system, intended to provide a more realistic picture of the consequences of the bifurcation.

We consider first a 1-dimensional landscape, consisting of a spot of high carrying capacity $\left(K>K_{c}\right)$ in the middle of a bigger region of low carrying capacity $(K<$ $K_{c}$ ). A typical situation is shown in Fig. 4 , where vertical lines represent the boundaries between the three zones. From an arbitrary initial condition of the populations, a steady state is attained in which the infected population is concentrated at the spot of higher $K$, that constitutes a "refugium." A "leak" of infection is seen outside the high- $K$ region, due to the diffusion. Far from this, the mouse population remains effectively not infected.

In Fig. 同 we show the steady state of a 2 -dimensional realization of the system (5),6) on a square grid which simulates a hypothetical landscape by assigning different values to $K_{i j}$, the carrying capacity at each site. This is supposed higher along a "river" as can be inferred from the density plots shown. The non-infected population occupies the whole landscape, with a non-homogeneous density. Moreover, as expected from the results of the homogeneous model, for small and moderate values of the diffusion coefficient, the infected population survives in a patchy pattern, only in the regions of high carrying capacity, becoming extinct in the rest. These "islands" of infection become reservoirs of the virus [6] or "refugia" [7], which are the places of highest risk for human exposure and contagion of the virus. It is also from these refugia that the disease would spread (blurring the patchiness, as observed in 3, 11]) when environmental conditions change. While our model is qualitative at this 

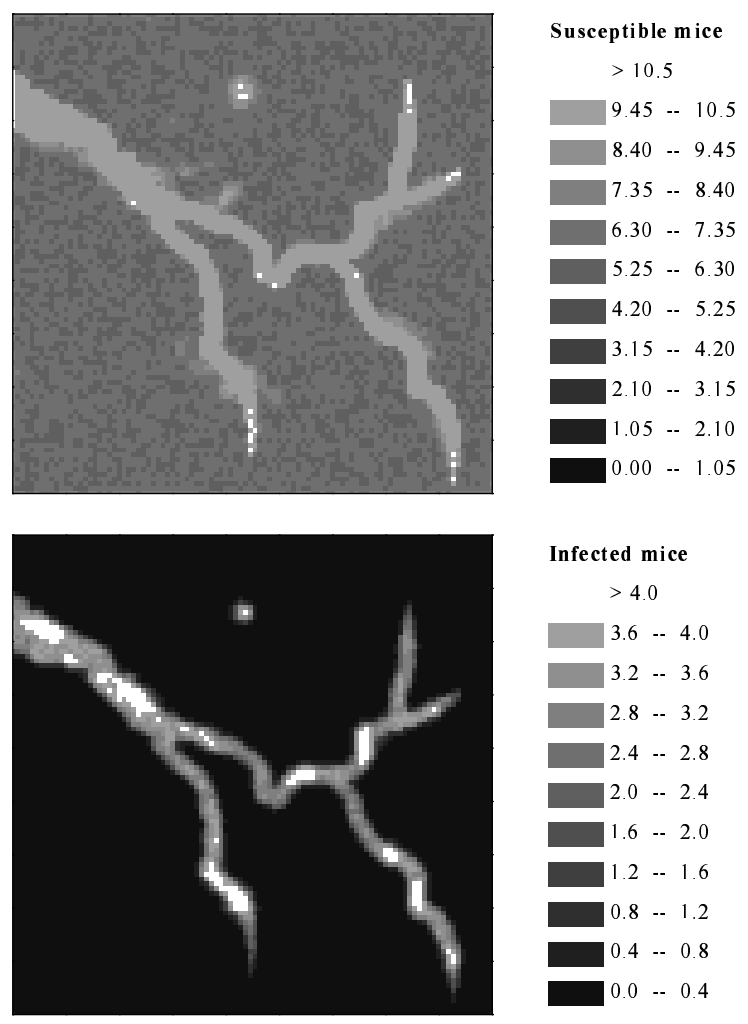

FIG. 5: Stationary solution of the extended model in two dimensions. The carrying capacity $K$ simulates a landscape where it is higher near a "river." Model parameters as in Fig. 1, $D=1$.

stage, this is precisely what is observed in the field. We comment in passing that the steady state distribution of neither infected nor susceptible mice reproduces exactly the distribution of the carrying capacity. This is the result of the interaction of diffusion with the nonlinear interactions. Thus, notice in the 1-dimensional representation shown in Fig. 1 that, although the carrying capacity follows a step distribution, the mice populations are not steps. Both $M_{S}$ and $M_{I}$ have diffusive "leaking" , the former exhibiting a dip as one moves out of the region of large capacity. Similarly, in the 2-dimensional case shown in Fig. 5 , we see that the peaks of the populations represented by pure white appear at different places for the susceptible and infected. They do not occupy the entire "river" region or follow precisely the peaks of the distribution of the carrying capacity.

\section{CONCLUDING REMARKS}

Two observed characteristics of Hantavirus infection have served as the focus of our present investigation: temporal patterns in the evolution of the population of infected mice, and emergence of spatial features in the landscape of infection, the so-called "refugia." Our theoretical model, represented by (5.6), incorporates nonlinear terms describing infection transfer between mice populations, a logistic description of their interactions with the environment, and diffusive terms representing their motion over the terrain. We have shown that the combination of these various terms, while simple, naturally predicts the temporal and spatial patterns whose observations have motivated the analysis. Our tools of investigation comprise of analytic stability considerations which result in features such as bifurcation behavior (e.g., Fig. 1) as well as numerical procedures which yield the temporal evolution (e.g., Fig. 2). The spatial extension inherent in our model allows us to analyze the dispersion relation describing in a simplified case departures from stationary states (see Fig. 3) and to deduce more generally the existence of the "refugia" (see Figs. 田 E).

We are currently in the process of investigating a number of further features of the spread of infection on the basis of the model and techniques explained in the present paper. They include among others: traveling waves which can depict the spread of fronts of infection emanating from the refugia in periods favorable to the propagation of the infection; situations in which the mice are limited in their meanderings to more or less localized regions for territorial reasons but spread the infection when the localized regions overlap; non-diffusive effects in the motion of the mice over the terrain; the effect of stochastic disturbances in the environment; and relevant details of the infection process such as delay effects related to finite incubation periods. The results of these investigations will be reported elsewhere.

\section{Acknowledgments}

We acknowledge many discussions with Terry Yates, Bob Parmenter, Fred Koster and Jorge Salazar from which we learnt much regarding the peculiarities of the hantavirus including the observation of refugia. We also thank Greg Glass, Karl Johnson and Luca Giuggioli for discusions. V. M. K. acknowledges a contract from the Los Alamos National Laboratory to the University of New Mexico and a grant from the National Science Foundation's Division of Materials Research (DMR0097204). G. A. thanks the support of the Consortium of the Americas for Interdisciplinary Science and the hospitality of the University of New Mexico. 
[1] C. Schmaljohn and B. Hjelle, Emerging Infectious Diseases 3, 95 (1997).

[2] J. N. Mills, T. L. Yates, T. G. Ksiazek, C. J. Peters and J. E. Childs, Emerging Infectious Diseases 5, 95 (1999).

[3] J. N. Mills, T. G. Ksiazek, C. J. Peters and J. E. Childs, Emerging Infectious Diseases 5, 135 (1999).

[4] C. H. Calisher, W. Sweeney, J. N. Mills and B. J. Beaty, Emerging Infectious Diseases 5, 126 (1999).

[5] C. A. Parmenter, T. L. Yates, R. R. Parmenter and J. L. Dunnum, Emerging Infectious Diseases 5, 118 (1999).

[6] A. J. Kuenzi, M. L. Morrison, D. E. Swann, P. C. Hardy and G. T. Downard, Emerging Infectious Diseases 5, 113 (1999).

[7] Terry L. Yates, personal communication (2001).

[8] R. M. Anderson and R. M. May, Infectious diseases of humans, Dynamics and control (Oxford University Press,
Oxford, 1992).

[9] J. D. Murray, Mathematical Biology, 2nd ed. (Springer, New York, 1993).

[10] C. R. Terman, in Biology of Peromyscus (Rodentia), J. A. King (editor) (The American Society of Mammalogists, Special publication No. 2, 1968).

[11] K. D. Abbot, T. G. Ksiazek and J. N. Mills, Emerging Infectious Diseases 5, 102 (1999).

[12] G. E. Glass et al., Emerging Infectious Diseases 6, 238 (2000).

[13] L. F. Stickel, in Biology of Peromyscus (Rodentia), J. A. King (editor) (The American Society of Mammalogists, Special publication No. 2, 1968).

[14] S. H. Vessey, American Zoologist 27, 879 (1987). 